\title{
Android-based Navigation System for Elderly People in Hospital
}

\author{
Ryoji Aoki, Hiroshi Yamamoto, Katsuyuki Yamazaki \\ Nagaoka University of Technology, 1603-1 Kamitomioka, Nagaoka, Niigata 940-2188 Japan \\ e-mail: aokir@stn.nagaokaut.ac.jp
}

\begin{abstract}
With the increase in the number of elderly people, a guidance system that can be used for medical examination in hospitals becomes to attract attention. As a position estimation technology for indoor guidance, a Wi-Fi positioning system has been studied. However, Wi-Fi radio waves are not available in the hospitals because that affect operations of medical equipment. In this paper, we propose a new realtime indoor guidance system using Personal Handy-phone System (PHS) and Android device for visitors in hospitals. Our proposed system utilizes a combination of Received Signal Strength Indication (RSSI) of PHS and Dead Reckoning based on sensors of an Android device in order to estimate a user's indoor position without affecting operation of medical equipment. Through the experimental evaluation, we have clarified that the proposed system has successfully guided subjects to the destinations with accuracy of 93.3[\%].
\end{abstract}

Keywords - Indoor Position Estimation, PHS, RSSI, Dead Reckoning, Android

\section{INTRODUCTION}

In a large hospital consisting of many medical departments, it is difficult for visitors to reach their destinations smoothly. In particular, in case of a medical examination, visitors often get lost because they should visit many medical departments that are apart from each other. This causes a serious problem particularly for elderly people. To solve this problem, a guidance system that can be used in hospitals is attracting attention. In the guidance system, indoor position estimation is one of the most important technologies.

As the indoor positioning technology, Dead Reckoning has been widely studied [1], [2], [3]. In the Dead Reckoning, a user's movement is estimated by analyzing data obtained from various sensors (e.g., acceleration, gyroscope, etc.). Especially, in recent years, Android phones become the best candidate of the sensing device because they are equipped with a wide variety of sensors. Furthermore, as an indoor position estimation technology, a wireless positioning system using Wi-Fi [4], [5] has been studied. However, Wi-Fi access points are not available in the hospitals because $2.4 \mathrm{GHz}$ radio waves affect operations of medical equipment. On the other hand, an indoor Personal Handy-phone System (PHS) is commonly used for constructing inter-office telephone in the hospitals. This is because a PHS device does not use the $2.4 \mathrm{GHz}$ Industry-Science-Medical (ISM) radio bands and its maximum transmission power is much lower than a cell phone [6].

Therefore, this paper proposes a new realtime guidance system for hospitals using a PHS and an Android device. The Android device estimates user's position based on the Received Signal Strength Indication (RSSI) of PHS radio waves in addition to Dead Reckoning with acceleration, compass and gyroscopes sensor, and then displays route guidance. A user-friendly interface on the Android is also proposed, which can be easily understood even for elderly people. Furthermore, the effectiveness of the proposed system is evaluated through route guidance experiments.

\section{RELATED WORKS AND OBJECTIVES OF THIS STUDY}

\section{A. Issue of Guidance in Hospital}

In Japan, elderly people are rapidly increasing [7] and their health-care becomes a critical social cost. Medical examination at an early stage is very important to detect a sign of diseases, hence will decrease medical cost for afterwards surgical operation, hospitalization, etc. On the other hand, there are many inspection items more than 10 in the medical examination and the inspection items are expected to increase further with advance in medical technology.

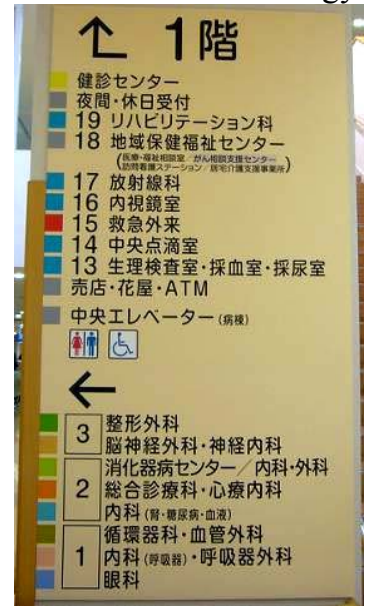

Figure 1. A lot of destinations listed on guidance board in a hospital

Figure 1 represents a typical guidance board in a hospital. As shown in this figure, the hospital consists of many medical departments and examinees should visit a lot of examination rooms (e.g., C.T. scan, MRI, endoscopy, etc.) that are apart 
from each other. For this reason, route of medical examination is complicated and guidance in a hospital is an important issue. However, automated guidance which can lead visitors to an appropriate place at an appropriate time has not been established yet although IT is utilized for various activities (e.g., Electronic Health Record) in hospitals.

\section{B. Existing Studies}

The position estimation system using a GPS has become available with a wide spread of mobile devices equipped with GPS. However, the GPS cannot be used in indoor environment because GPS signals are blocked by walls or ceilings.

As indoor positioning technologies, methods using radio waves transmitted from multiple transmitters have been researched and developed. In these systems, signal strength of radio waves of Wi-Fi [8] or Bluetooth [9] is used. However, these radio waves use the ISM bands, which may interfere with medical equipment, and hence must not be used in hospitals.

On the other hand, PHS can be used for inter-office telephone in the hospital because the impact on medical equipment is very small [10]. Furthermore, as shown in Table 1 , the output power of PHS is small compared with a cellular phone. An outdoor positioning method using radio waves of PHS has already been studied [11]. However, the practicality of PHS radio waves for indoor guidance system has not been investigated.

TABLE 1. OUtPut POWER OF EACH TELECOMMUNICATIONS TECHNOLOGIES

\begin{tabular}{|l|l|}
\hline \multicolumn{1}{|c|}{$\begin{array}{c}\text { Telecommunications } \\
\text { Technology }\end{array}$} & \multicolumn{1}{c|}{$\begin{array}{c}\text { Burst Average } \\
\text { Power }[\mathbf{m W}]\end{array}$} \\
\hline PHS & 80 \\
\hline IMT-2000(3G) & 200 or 250 \\
\hline
\end{tabular}

Furthermore, a positioning technology using sensors on the Android device has been studied [12]. Position estimation using various motion sensors is called Dead Reckoning. The Dead Reckoning does not generate radio wave, hence the indoor position estimation method using the Dead Reckoning is a useful tool in the hospital. However, in the Dead Reckoning, error of the sensor data is accumulated with time [13], hence, correction of the accumulated error is one of the important issues.

\section{Objectives of This Study}

Considering these situations, we propose a guidance system which utilizes a combination of RSSI of PHS and Dead Reckoning using sensors of Android devices. In this study, acceleration, compass and gyroscope sensors on the Android device have been utilized for estimating the user's position by the Dead Reckoning. The aim of our study is to develop a guidance system on an Android device including smart user interface which can be used by elderly people.

\section{III.PROPOSED SYSTEM}

\section{A. Overall structure of Proposed System}

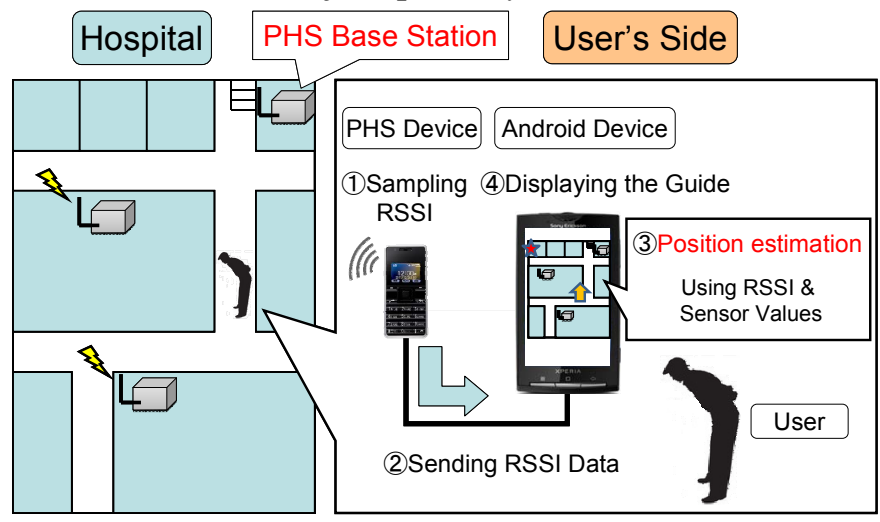

Figure 2. Overview of the guidance system in a hospital using PHS

Figure 2 shows an overview of the proposed guidance system in the hospital. This system provides a route guidance interface which is easy even for elderly users to understand. The procedures of the system are given as follows.

1. A PHS device samples RSSI of radio waves from PHS base stations (PBS).

2. The RSSI is transmitted from the PHS device to an Android device through USB.

3. The Android device estimates the user's position based on the RSSI and Dead Reckoning using sensors.

4. The Android device displays a guidance of the user's position to the destination.

In this study, it is assumed that the user holds an Android device and a small PHS device, which are received at a reception desk of the hospital. Although there's a small and compact PHS device in the current market, it can be a chip when this system becomes a commercial product. In addition, a PHS smart phone operated by Android OS has already been available on the market and it can make the proposed system more convenient.

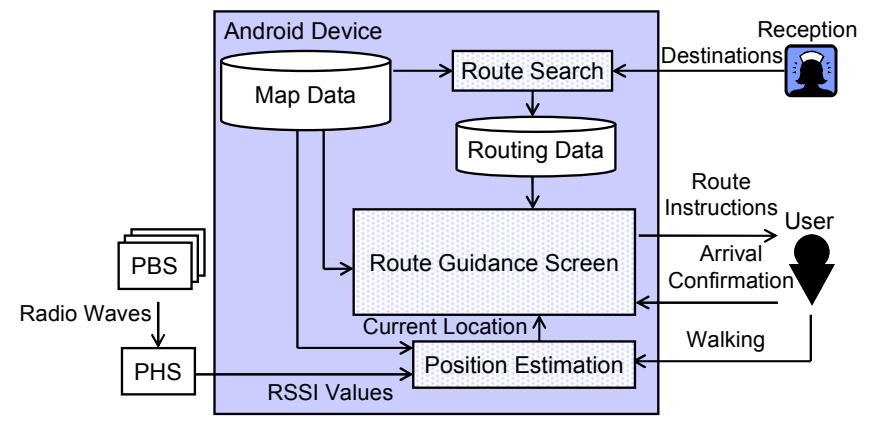

Figure 3. Block diagram of guidance system in a hospital

Figure 3 represents a block diagram of the guidance system. The map data consists of coordinates of nodes and links between adjacent two nodes. Coordinates of the nodes correspond to the corners, intersections and consultation rooms. The map data also includes positions of PBS and RSSI values at each point. In addition, it is assumed that hospital 
officials set destinations (e.g., X-ray examination room) at a reception desk for a visitor.

\section{B. Position Estimation using Dead Reckoning and PHS}

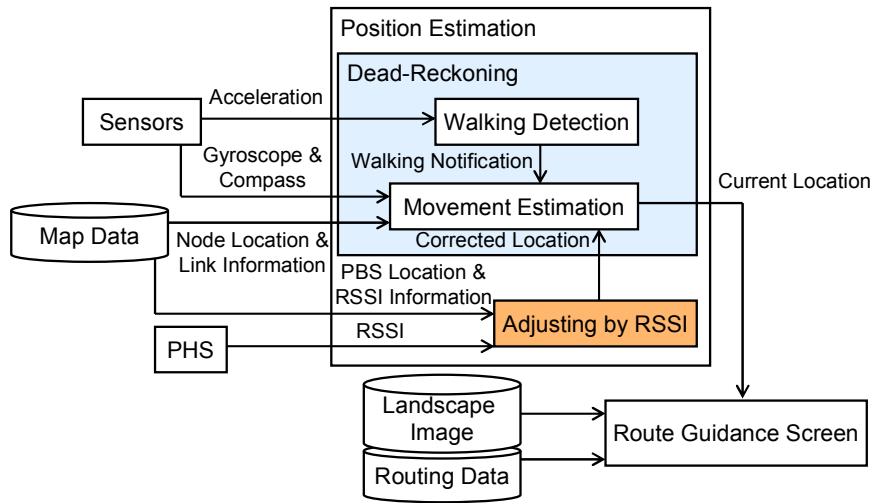

Figure 4. Block diagram of position estimation

A block diagram of position estimation is illustrated in Figure 4. Dead Reckoning is carried out by utilizing acceleration, compass and gyroscope sensors on the Android device. Accumulated error of user's location that is estimated by the Dead Reckoning is corrected by using the RSSI of PHS. Then, the estimated user's current location is utilized for the Route Guidance.

\section{1) Dead Reckoning:}

Dead Reckoning is a method of estimating the user's position based on the sensor data. In the Dead Reckoning, the position change is estimated by detecting user's movements and changes of the moving direction of the user from the sensor data. The procedure of the Dead Reckoning is composed of the following ways.

1. The number of steps during walking is detected by an acceleration sensor.

2. A moving distance is calculated by multiplying the number of steps and the user's stride length.

3. Direction of movement is detected by compass sensor.

4. Turning direction at a corner is estimated using a gyroscope sensor values.

5. The estimated position is adjusted to coordinates of the corner when the change of the moving direction is detected in order to correct error.

In this study, we use an Android phone "Galaxy Nexus" that is equipped with these sensors for the Dead Reckoning. In addition, a subject is assumed to hold the Android phone as its screen can be seen. Figure 5 is a snapshot of a subject using the proposed application.

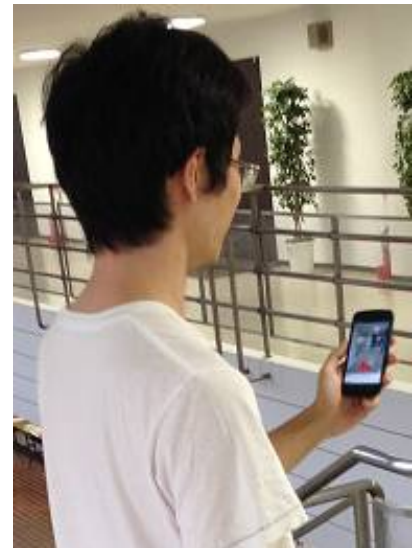

Figure 5. Snapshot of a subject when using the proposed application

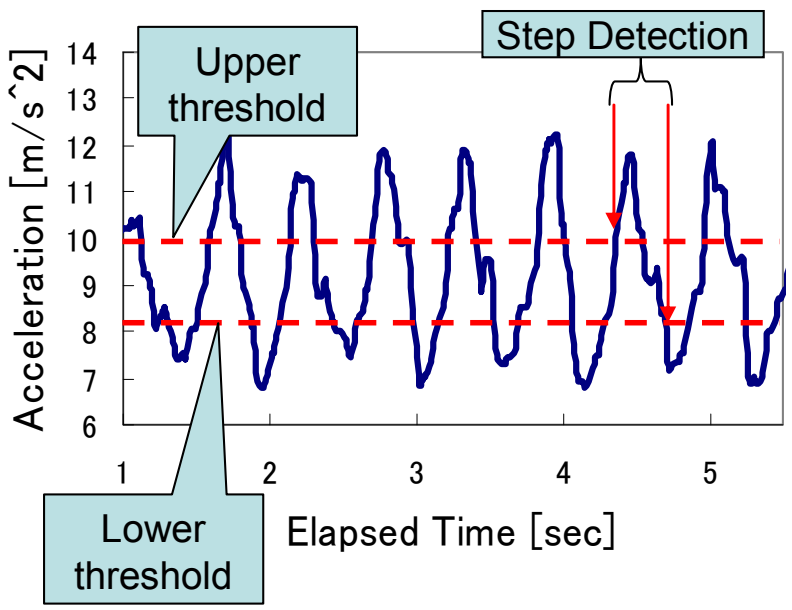

Figure 6. 3-axis acceleration during walking

Figure 6 shows 3-axis acceleration data during walking. It is clear from the figure that acceleration sensor values vary step by step. As shown in this figure, we set two threshold values so that user's walking can be detected. The system judges that the user walks one step when the acceleration value is less than the lower threshold after exceeds the upper threshold. In proposed application, value of the upper threshold is set to $9.99\left[\mathrm{~m} / \mathrm{s}^{2}\right]$ and the lower threshold is set to $8.17\left[\mathrm{~m} / \mathrm{s}^{2}\right]$.

Next, the moving direction of the user is to be detected. In the proposed application, the user is assumed to stay on the straight line between nodes. Thus, the application detects whether the user goes forward or backward on the path by using the value of a compass sensor. And then, an estimated position of the user is moved stride length toward the estimated moving direction. 


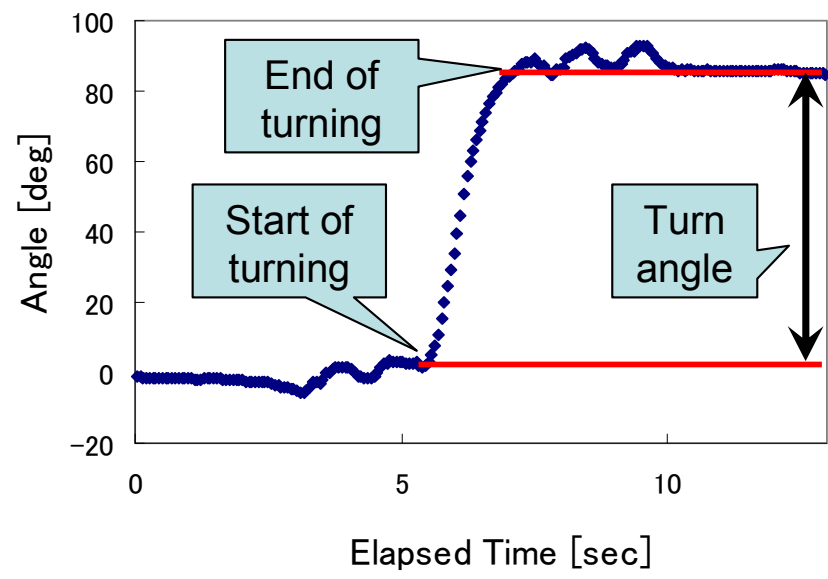

Figure 7. Gyroscope sensor values during turning

Gyroscope sensor values during turning are illustrated in Figure 7. From the previous study [14], it is observed that the value of the gyroscope sensor has less noise of walking and more accurately catches up the turning than the compass sensor. Therefore, the application uses the gyroscope sensor for turning detection.

In the turning detection, we focus on the variation of the gyroscope sensor values while walking. The application decides to detect the start of turning when the angle continues to change more than 10 degrees. Then, if the angle changes only less than 10 degrees during 3 walking steps, it is considered that the turning is ended.

When the turning is detected, the application searches the nearest corner to the estimated user's position from the map data, and the estimated position is adjusted to coordinates of the corner. This process is called a corner matching. By the corner matching, it becomes possible to correct estimation errors of the current position. Finally, the application determines next moving direction by checking turning angle.

\section{2) PHS Based Indoor Location Estimation:}

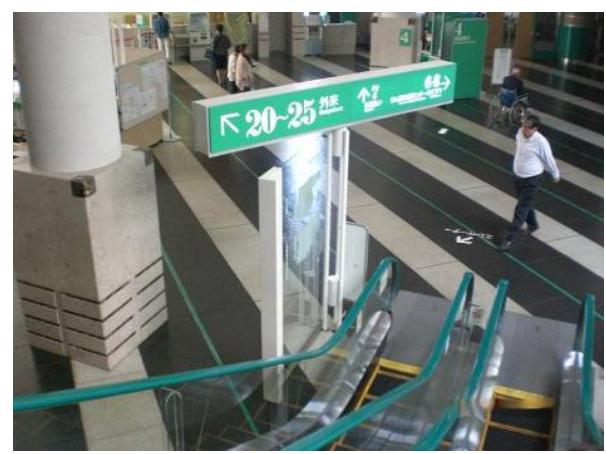

Figure 8. Open space and escalators in a hospital

The position estimation using the Dead Reckoning has weakness when it is used in an open space and escalators of a hospital. As shown in Figure 8, escalators are often deployed in a large hospital. While a user is taking an escalator, the Dead Reckoning is not available because the sensors cannot catch any movement of the user.
Moreover, the Dead Reckoning has a problem in terms of the accumulated error. In the Dead Reckoning, an impact of accumulated error appears when walking distance becomes longer in the same direction, because actual stride length of the user varies step by step. It is possible to correct the accumulated error by corner matching. However, when multiple intersections exist in the moving direction, the corner matching may select a wrong position. Furthermore, in the open space as shown in Figure 8, the corner matching is difficult because the moving direction of the user can be changed anywhere.

In order to solve these problems, we propose to use RSSI of PHS in this study.

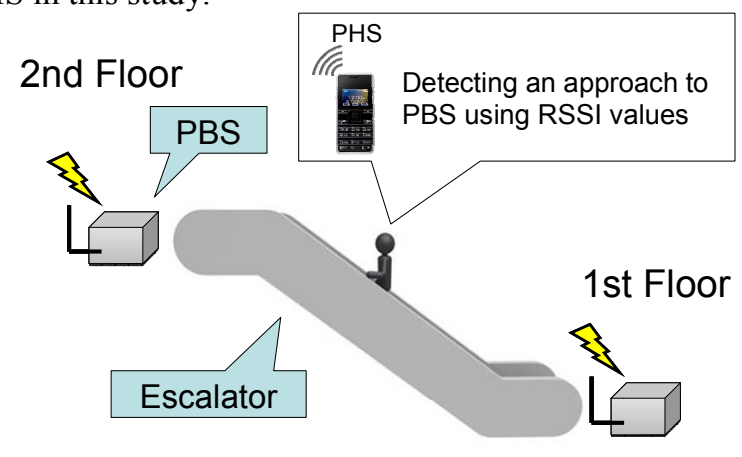

Figure 9. Use of RSSI of PHS for detection of user's position

Figure 9 shows a use of RSSI of PHS for detection of user's position. Here, PBSs are deployed on each of floors and a user has a PHS device. The proposed application detects an approach to the PBS by referring to RSSI values measured by the PHS device, and changes the user's estimated position to the location nearby the PBS. Likewise, it is possible to correct the accumulated error in open space by utilizing PBS deployed in such doorway.

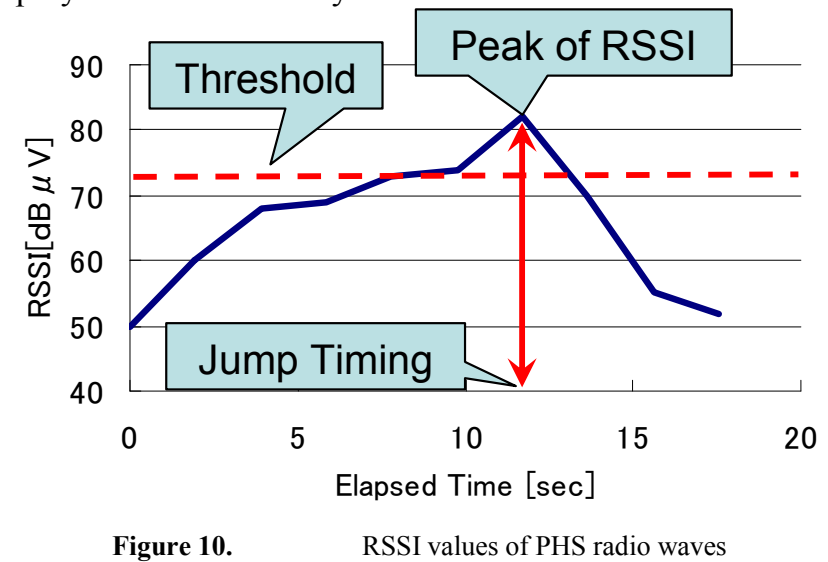

RSSI values of PHS radio wave are illustrated in Figure 10. When the user accesses the PBS, the RSSI value exceeds the threshold and becomes peak. Thereby, the estimated position jumps to coordinates of the PBS point. In the proposed application, the threshold is set to $72[\mathrm{~dB} \mu \mathrm{V}]$.

\section{User Interface}

The Route Guidance Screen displays information for guiding a visitor from the estimated position to the destination. 
User interface of the guidance should be friendly for elderly people who are main visitors of a hospital. Therefore, we adopt Turn by Turn method [15] as a UI of the guidance display. The Turn by Turn method guides the user to a destination by indicating the moving direction at each corner. Compared with the method displaying the route on a map image, the Turn by Turn method has merits that the instructions are intuitive and easy to be understood. We propose to use two types of Turn by Turn UI depending on user's situation.

\section{1) Starting Guidance:}

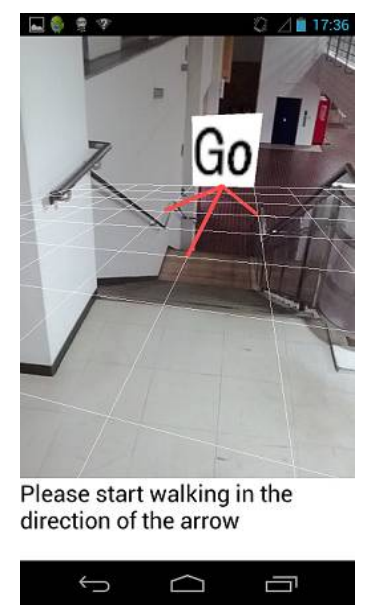

Figure 11. Guidance method - 1: Start moving direction

When the guidance is started, the UI shows the moving direction to the user by drawing an arrow on a camera preview as illustrated in Figure 11. At this time, a compass sensor detects moving direction and a position of Android device is calculated from acceleration sensor values in order for the UI to draw an arrow image so as to indicate the accurate moving direction. Besides, the UI switches to another UI when the user walks 3 steps in the direction of the arrow.

\section{2) Guidance for Turning Corner:}

Except for starting time, the application shows moving direction by displaying an indication message and guidance arrow on a previously captured image. When it detects turning at a corner, guidance image at the next corner will be displayed.

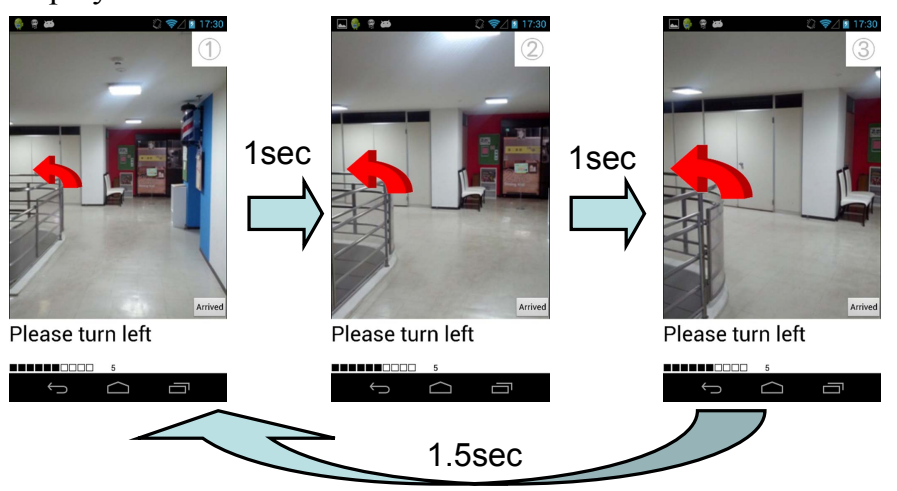

Figure 12. Guidance method - 2: Turning a corner
Figure 12 represents an example of the guidance UI. In order to avoid confusing users at a point where there exist many corners, the application shows animation including three images.

\section{Route Search}

In the proposed application, Dijkstra's algorithm [16] is utilized to automatically determine a route between the estimated user's position to the destination. By using the algorithm, the shortest path can be derived at low cost.

\section{EVAluation OF Route GUIDANCE APPLICATION}

\section{A. Outline of Experiment}

In order to evaluate the performance of the proposed guidance application, we have conducted route guidance experiments. In this study, experiments were performed in buildings of Nagaoka University of Technology. The details of the experiments are given as follows.

- 5 subjects move to their destinations according to guidance UI of the application.

- Each subject walks 3 routes.

- 6 points on the map are assumed as medical examination points (e.g., C.T. scan room), and a start point is assumed as Reception.

- Each route includes all 6 medical examination points, and the final destination is the start point.

- The application detects arrival of the user when the subject presses the "Arrival Button".

- Stride of each subject is calculated in advance from the number of steps when walking a corridor of $40 \mathrm{~m}$.

In order to evaluate correction method using the RSSI of the PHS, we have conducted experiments using two types of position estimation methods (Dead Reckoning only and Dead Reckoning and PHS).

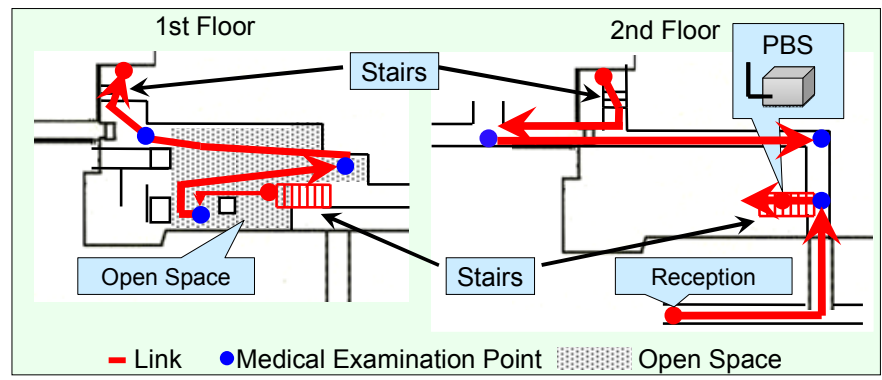

Figure 13. The map data of the Nagaoka University of Technology that represent a hospital

The map data of the experimental environment is represented in Figure 13. The arrows indicate Route 2 in Table 2. As shown in this figure, the first floor of the building is an open space, and a PBS was deployed on a landing of the stairs. 


\section{B. Experiment Result}

Evaluation result of the proposed route guidance system is given in Table 2.

TABLE 2. RESUlt OF ROUTE GUIDANCE EXPERIMENT

\begin{tabular}{|c|c|c|c|c|}
\hline \multirow{2}{*}{ Route } & \multirow{2}{*}{ Subjects } & $\begin{array}{c}\text { Medical } \\
\text { examination } \\
\text { point }\end{array}$ & & \multicolumn{2}{c|}{ Guidance success rate[\%] } \\
\cline { 4 - 5 } & & 6 & 60.0 & 100 \\
\hline 1 & 5 & 6 & 60.0 & 100 \\
\hline 2 & 5 & 6 & 100 & 80.0 \\
\hline 3 & 5 & 73.3 & 93.3 \\
\hline \multicolumn{3}{|c|}{ Average only } & DR \& PHS \\
\hline
\end{tabular}

When the method uses the Dead Reckoning only, the guidance success rate was $73.3[\%]$. On the other hand, the position estimation using the Dead Reckoning and the RSSI of PHS achieved a high success rate of $93.3[\%]$. This is because the Dead Reckoning cannot decide the correct one from multiple nodes on the same corridor, while the estimated position can be corrected by detecting that the subject had passed in front of the PBS from the RSSI values. These results suggest that the position correction by RSSI of PHS is effective.

The method using the Dead Reckoning and PHS could not complete the guidance in Route 3. This was caused by the case when the stride length of a subject became longer than the premeasured one. Therefore, we will develop an automatic adjustment method of user's stride in the future study.

\section{Conclusions}

This paper has proposed a realtime guidance system in a hospital using PHS and Android device. Specific feature of this system is a use of the combination of Dead Reckoning based on sensor data and RSSI of PHS. The Dead Reckoning is basically used to estimate user's indoor location, and accumulated error of the estimated location is corrected by utilizing the PHS radio waves, which have less impact on the medical equipment. Furthermore, a new intuitive user interface based on the turn by turn method has been proposed.

In order to evaluate effectiveness of the proposed guidance application, we have conducted route guidance experiments in Nagaoka University of Technology campus. Evaluation result of the developed system has shown that the position estimation using the Dead Reckoning and the RSSI of PHS achieves a high success rate of $93.3[\%]$. The results indicate that the combination of the RSSI of PHS and the Dead Reckoning realizes accurate route guidance in a hospital.

In the future study, we'll further study to develop a dynamic estimation method of user's stride for improving the guidance success rate.

Authors would like to express sincere thanks to Mr. T.Hiyama, K.Iguchi and K.Kasai of Abit Corporation for their technical support on PHS technologies.

\section{REFERENCES}

[1] K. Sagawa, K. Koiwa, M. Susumago, H. Inooka, "Estimation of an indoor 3D walk course by acceleration integration", 22th Joint Conference on Medical Information Japan Journal of Medical Informatics 22, pp.242-243, Nov. 2002.

[2] M. Yamamoto, E. Kamioka, "Location Estimation System using Acceleration Sensor", The Institute of Electronics, Information, and Communication Engineers Technical Report, Mobile Network and Applications, vol.110, no.40, pp.139-144, May. 2010.

[3] M. Kourogi, T. Kurata, "Personal Positioning Based on Walking Locomotion Analysis with Self-Contained Sensors and a Wearable Camera", in Technical Report of The Institute of Electronics, Information, and Communication Engineers Technical Report, PRMU 103(737), pp.25-30, Mar. 2004.

[4] N. Kawaguchi, "Locky.jp: Wireless LAN Position Estimation and Its Application", The Institute of Electronics, Information, and Communication Engineers Technical Report, ITS vol.107, no.161, pp.1-4, Jul. 2007.

[5] Y. Yokota, T. Hitoyasu, M. Miki, H. Yokouchi, M. Yoshimi, "Location Estimation in Indoor Environment based Distribution of the RSS", in Proc. the Annual Conference of The Japanese Society for Artificial Intelligence, vol.24, no.3C2-2, pp.1-4, Jun. 2010.

[6] Research Committee on the use of radio waves for regional development, "Research Report on Telecommunications in the Hospital", p.8, Mar. 2002

[7] "Daitoshi Iryo Crisis 1.", NHK ONLINE, Dec. 20, 2012 http:/www.nhk.or.jp/ohayou/marugoto/2012/12/1220.html , accessed Nov.7, 2013.

[8] K. Anzai, S. Okajima, H. Tsubokawa, "The estimate of the indoo position that used a smartphone and the suggestion of the walk navigation system", in Multimedia, Distributed, Cooperative, and Mobile Symposium 2011, pp..921-927, July 2011

[9] T. Takakai, M. Fujii, Y. Watanabe, A. Ito, "A Study on Location Awareness System using cellular phone with Bluetooth", The Institute of Electronics, Information, and Communication Engineers Technical Report ITS, vol.108, no.205, pp.31-36, Sep. 2008

[10] Medsafe.Net. "Utilizing PHS for communication tools in hospital", Medsafe.Net, Nov.21, 2006 http://www.medsafe.net/contents/recent/107jikeikai.html, accessed Mar.26, 2013.

[11] Y. Uramoto, S. Kitamura, "Examination of simplified position information acquisition depending on PHS utilization", in Proc. The Institute of Electronics, Information, and Communication Engineers General Conference, no.B-5-138, p.525, Mar. 1997.

[12] H. Ookura, H. Yamamoto, K. Yamazaki, "Development and evaluation of walking path estimation system using sensors of Android device and vector map matching", in Proc. The Institute of Electrical and Electronics Engineers, The International Conference on Information Networking.2012, pp.25-29, Feb. 2012.

[13] K. Katsuhiko, N. Kawaguchi, "Gate-Passing Detection Method Based on WiFi Significant Points", in Proc. of the World Congress on Engineering 2013, Voll.II, pp.1409-1414. July 2013.

[14] R. Aoki, H. Yamamoto, K. Yamazaki, "Realtime Guidance System in Hospital using PHS and Android Device", in Proc. The Institute of Electrical and Electronics Engineers, Computer Software and Applications Conference 2013, pp.451-454, July 2013.

[15] H. Ito, "The Status of the Navigation and Route Guidance System in Foreign Countries and Their Standardization Activities", The International Association of Traffic and Safety Sciences Review, Vol.26, No.4, pp.232-242, Sep. 2001.

[16] K. Kato, "Note on Optimal Path for an Electric Vehicle", The Institute of Electronics, Information, and Communication Engineers Technical Report, COMP vol.109 (465), pp.13-17, Mar. 2010. 


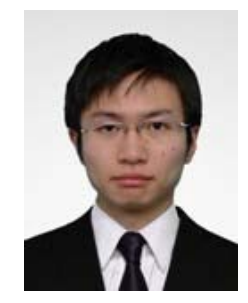

Ryoji Aoki received B.E. degree from Nagaoka University of Technology in '12. He is currently a graduate school student in Nagaoka University of Technology. His research interests include computer networks and mobile network system.

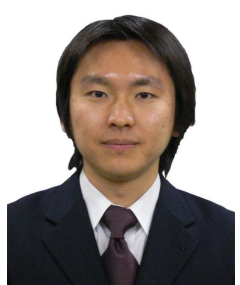

Hiroshi Yamamoto received M.E. and D.E. degrees from Kyushu Institute of Technology, Iizuka, Japan in

'03 and '06, respectively. From April '06 to March '10, he worked at FUJITSU LABORATORIES LTD. Kawasaki, Japan. Since April '10, he has been an Assistant Professor in the Department of Electrical Engineering, Nagaoka University of Technology. His

research interests include computer networks, a member of the IEEE. distributed applications, and networked services. He is

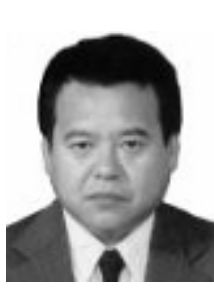

Technology.

Katsuyuki Yamazaki received B.E. and D.E degrees

from the University of Electro-communications and Kyushu Institute of Technology in '80 and '01, respectively. At KDD Co. Ltd., he had been engaged in R\&D and international standardization of ISDN, S.S. No.7, ATM networks, L2 networks, IP networks, mobile and ubiquitous networks, etc., and was responsible for R\&D strategy of KDDI R\&D Labs. He is currently a Professor of Nagaoka University of 